\title{
Pathologic Stage II Esophageal Squamous Cell Carcinoma AJCC v8
}

National Cancer Institute

\section{Source}

National Cancer Institute. Pathologic Stage II Esophageal Squamous Cell Carcinoma A/CC

v8. NCI Thesaurus. Code C133532.

Stage II includes: IIA: (T2, N0, M0, G2-3, Tumor location: Any); (T2, N0, M0, GX, Tumor location: Any); (T3, N0, M0, Any G, Tumor location: Lower); (T3, N0, M0, G1, T umor location: Upper/middle); IIB: (T3, N0, M0, G2-3, Tumor location: Upper/middle); (T3, N0, M0, GX, Tumor location: Any); (T3, N0, M0, Any G, Tumor location: Unknown); (T1, N1, M0, Any G, T umor location: Any). T2: Tumor invades the muscularis propria. T1: Tumor invades the lamina propria, muscularis mucosae, or submucosa. T3: Tumor invades adventitia. N0: No regional lymph node metastasis. N1: T umor with metastasis in one or two regional lymph nodes. M0: No distant metastasis. GX: Grade cannot be assessed. G1: Well-differentiated. G2: Moderately differentiated. G3: Poorly differentiated, undifferentiated. (AJCC 8th ed.) 